\title{
RESPONSE VARIATION OF CHLADNI PATTERNS ON VIBRATING ELASTIC PLATE UNDER ELECTRO-MECHANICAL OSCILLATION
}

\author{
A. E. Ikpe ${ }^{1, *}$, A. E. Ndon ${ }^{2}$ and E. M. Etuk ${ }^{3}$ \\ 1, DePt of Mechanical Engineering, University of Benin, P.M.B. 1154, Benin, Edo State, NIGERIA \\ 2, Dept of Civil Engineering, Akwa Ibom State University, MkPat Enin, Akwa Ibom State, NIGERIA \\ 3, DePt of Production Engineering, University of Benin, P.M.B. 1154, Benin, Edo StATE, NIGERIA \\ E-mail addresses: ${ }^{1}$ aniekan.ikpe@eng.uniben.edu, ${ }^{2}$ ibiamndon@outlook.com, ${ }^{3}$ alwaysetuk@gmail.com
}

\begin{abstract}
Fine grain particles such as sugar, sand, salt etc. form Chladni patterns on the surface of a thin plate subjected to acoustic excitation. This principle has found its relevance in many scientific and engineering applications where the displacement or response of components under the influence of vibration is vital. This study presents an alternative method of determining the modal shapes on vibrating plate in addition to other existing methods like the experimental method by Ernst Chladni. Three (3) finite element solvers namely: CATIA 2017 version, ANSYS R15.0 2017 version and HYPERMESH 2016 version were employed in the modelling process of the $0.40 \mathrm{~mm} \times 0.40 \mathrm{~mm}$ plate and simulation of corresponding mode shapes (Chladni patterns) as well as the modal frequencies using Finite Element Method (FEM). Result of modal frequency obtained from the experimental analysis agreed with the FEM simulated, with HYPERMESH generated results being the closest to the experimental values. It was observed that the modal frequencies obtained from the FEM and experimental approach increased as the excitation time increased. ANSYS R15.0 and HYPERMESH software clearly represented the modal lines and mode shapes for each frequency which CATIA software was somewhat limited. This study has shown that FEM is an effective tool that can save time and energy invested in acoustic experiments in determining modal frequencies and patterns.
\end{abstract}

Keywords: Vibration, Chladni patterns, Modal frequency, Thin plate, Experimental analysis

\section{INTRODUCTION}

Visualization of patterns formed on an acoustically excited thin plate under the influence of a vibrator can be achieved when fine sand, grains of sugar etc. are deposited on the plate surface during vibration. This great invention had its publicity in early 1787 when Ernst Chladni found that fine grain particles on the plate surface can rearrange themselves into aesthetically developed patterns [1,2]. Despite their aesthetics and artistic beauty, Chladni patterns have applications in vibration of flat plates, construction of musical instruments such as violins, guitars, speakers etc. $[3,4]$. Application of Chladni's method further indicate that plates with different shapes under the influence of vibration can present diverse patterns on the surface of the plate. The natural frequencies at which thin plates vibrate is vital to the visualization of nodal lines formed by the Eigen modes. The ideal method of analysing these patterns is by solving the inhomogeneous Helmholtz equation using proper boundary conditions. However, it is difficult and time consuming to accurately employ this method, particularly in the case of vibrating plates with irregular open boundaries [5]. For example, Amore [6] computed a method for solving the Helmholtz equation using mathematical relation known as "little sinc functions" which was only applicable to irregular and/or inhomogeneous membrane with fixed boundary conditions. According to Owunna et al. [7] estimating the natural frequencies of vibrating plate is an aspect of dynamic analysis, referred to as eigenvalue analyses. While the frequencies are

* Corresponding author, tel: +234-902-477-3812 
determined through experimental process, the resonant frequencies are acquired by probing the variation of the effective impedance of the oscillator with and without the thin plate. Using Qt framework and the Open Graphics Library, Muller et al. [8] developed the graphical user interface NumChladni, an interactive tool for studying arbitrary twodimensional vibrating plates. The eigenvalue system to determine the eigenmodes of arbitrarily shaped, thin plates based on Finite Element Method (FEM) was derived. Shridhar [9] examined the effects of adhesion, friction, and damping on the development of Chladni patterns, and suggested actual applications for these effects. This was achieved through the application of spatial autocorrelation analysis in exploring the effects of adhesion and frictional forces on the rate of pattern formation. Investigation on how to control the motion of multiple objects on a Chladni plate revealed that the motion is sufficiently regular to be statistically modelled, predicted and controlled [10]. By playing carefully selected musical notes, the authors showed that the position of multiple objects can be controlled simultaneously and independently using a single acoustic actuator. This method allows the determination of independent trajectory, pattern transformation and sorting of multiple miniature objects in a wide range of materials. By means of ultrathin silicon membranes excited in the low ultrasound range, Vuillermet [11] illustrated that it is possible to form two-dimensional Chladni patterns of microbeads in liquid. From the findings, it was observed that the combined effects of an ultrathin plate excited at low frequency (yielding to subsonic waves) together with reduced gravity (arising from buoyancy) will enhance the importance of microstreaming in the Chladni problems. In this study, experiment was conducted at various frequencies to determine the corresponding Chladni patterns which were emulated using finite element method.

\section{MATERIALS AND METHOD}

For the experimental procedure, a square thin plate $(0.40 \mathrm{~mm} \times 0.40 \mathrm{~mm})$ was attached to the driver (indicated by the circular surface region in Figure 1a) by gently removing the screws from the oscillator surface and putting the screws back as shown in Figure $1 \mathrm{~b}$. This was followed by superimposing the square plate (see Figure 1c) on the surface of the oscillator, by ensuring that the centre hole of the plate fits into the central nub in the electromechanical oscillator.

To reduce friction, graphite powder was periodically applied on the plate surface and excesses were brushed off. This was performed before sprinkling grain particles of granulated sugar on the surface of the primary plate. The electro-mechanical oscillator was turned on, starting from a frequency signal of 100 Hertz at 7 seconds. The amplifier was slowly adjusted until vibration began to occur and the primary thin plate excited, producing line patterns while recording the modal frequencies. At intervals, the amplitude was reduced in order to sprinkle additional quantity of sugar on the plate. For each vibration phase, the frequency at which clear mode shapes formed were recorded accordingly. As the mechanical oscillator was driven with an amplified sinusoidal voltage, a digital galvanometer was connected in series with the mechanical oscillator to probe the effective current amplitude. This allowed the frequency response of the effective impedance of the mechanical oscillator to be measured. All investigations using FEM were performed on a $0.40 \mathrm{~mm} \times 0.40 \mathrm{~mm}$ CAD models of the thin plates in Figure 2 .

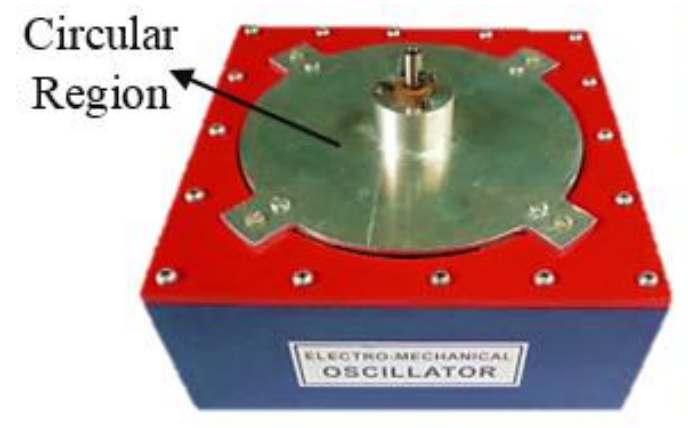

a

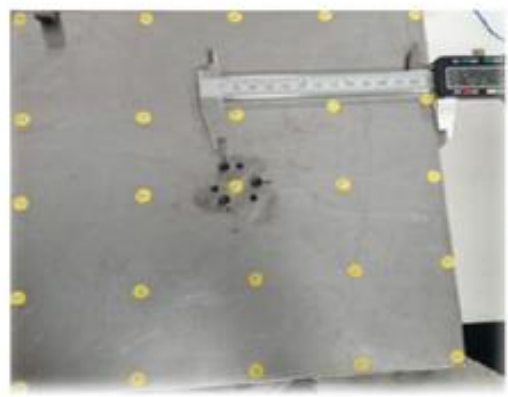

b

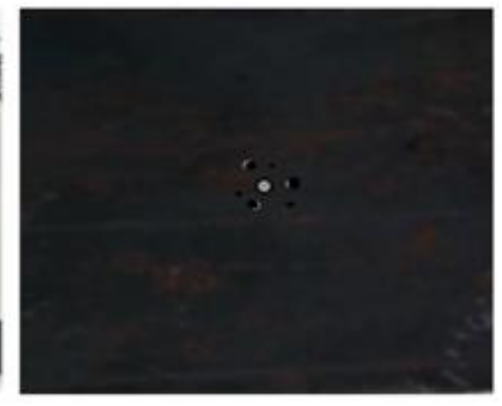

C

Figure 1: Experimental Set-up for the Plate Vibration 


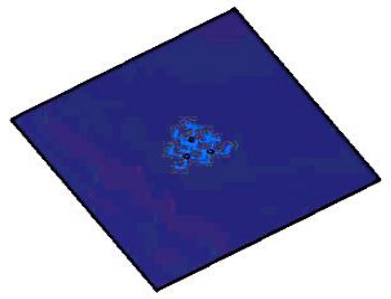

a. CATIA Plate Model

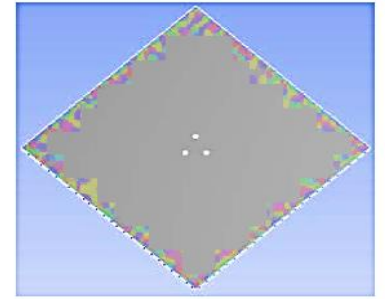

b. ANSYS Plate Model

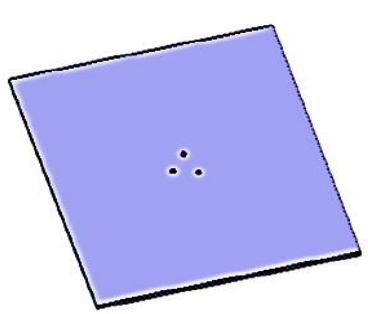

c. HYPERMESH Plate Model

Figure 2: CAD Models of the thin Plates

CATIA is an engineering and design software that enables the creation of 3D parts from 2D sketches, with functional tolerances as well as kinematic definition of points on simulated models. On the other hand, Ansys workbench performs similar function like CATIA, as the performance of designed products can be modelled, simulated and predicted with different specifications that correlate with experimental validation as demonstrated in this study. In addition, Ansys has an integrated solver that aids the application of loads and boundary conditions while Hypermesh is mainly designed for meshing of Computer Aided Design (CAD) parts. It helps breakdown the geometry or profile into smaller elements for effective application of the boundary conditions which in this study are the frequency signals ranging from $100 \mathrm{HZ}$ at $7 \mathrm{~s}, 14 \mathrm{~s}, 28 \mathrm{~s}, 35 \mathrm{~s}, 42 \mathrm{~s}$, $49 \mathrm{~s}$, and $56 \mathrm{~s}$ to $8888 \mathrm{~Hz}$ respectively. The following steps were adopted in the finite element method as applied to the mechanics of thin plates;

i. The thin plate models were constrained at the centre and in all degrees of freedom.

ii. Generation of 3D solid mesh. This divided the continuum into finite number of elements characterized by line segments and nodes.

iii. Selection of key points on the elements to serve as nodes where conditions of equilibrium and compatibility are applied.

iv. Assumption of displacement functions for individual element such that the displacements at each generic point is depended upon the nodal values.

v. Stiffness and equivalent nodal loads were established for a given element using flexibility method or energy principles.

vi. Equilibrium equations were generated for each set of nodes of the discritized continuum in terms of the idealized element.

vii. The equilibrium condition was solved for the nodal displacements.
Considering a thin flat plate driven by a timeharmonic source $F\left(r^{\prime}\right)$, the response function $\Psi(r$, $\left.r^{\prime}, \tilde{k}\right)$ of the vibrating plate can be solved with the inhomogeneous Helmholtz equation [12] given by Equation (1);

$$
\left(\nabla^{2}+\tilde{k}^{2}\right) \Psi\left(\mathrm{r}, \mathrm{r}^{\prime}, \tilde{k}\right)=F\left(r^{\prime}\right)
$$

Where, $\tilde{k}=k+i y, k$ is the driving wave number and $\mathrm{y}$ is the damping coefficient of the vibrating system. Equivalent deflections found for each mode shapes [13] is given in Equation (2);

$$
\begin{aligned}
V_{\text {max }}=\frac{D}{2} \iint_{A}\left\{\left(\frac{\partial^{2} W}{\partial x^{2}}+\frac{\partial^{2} W}{\partial y^{2}}\right)^{2}\right. \\
-2(1 \\
\left.-v)\left[\frac{\partial^{2} W}{\partial x^{2}} \frac{\partial^{2} W}{\partial y^{2}}-\left(\frac{\partial^{2} W}{\partial x \partial y}\right)^{2}\right]\right\} d x d y \\
=\frac{1}{2} K_{e f f}\left[W_{0}\left(x_{0}, y_{0}\right)\right]^{2}
\end{aligned}
$$

Where, $W_{0}\left(x_{0}, y_{0}\right)$ is the point on the square plate which has the maximum deflection and $\left(x_{0}, y_{0}\right)$ is the location of that point. The maximum deflection point differs for different mode shapes.

Under a time-harmonic condition, the effective coupling efficiency of the normalized power transferred from the point source to the vibrating plate can be given by Equation (3);

$$
\eta\left(r^{\prime} \bar{k}\right)=\left|\sum_{n} k_{n}{ }^{2} \cdot a_{n}\left(r^{\prime}, \bar{k}\right) \cdot \phi_{n}\left(r^{\prime}\right)\right|^{2}
$$

Where $k$ is the driving wavenumber, $k_{n}$ is the eigenvalues close to the driving wave number, $\phi_{n}\left(r^{\prime}\right)$ is the eigenmodes.

Applying the Greens function, the response function excited by any general source $f\left(r^{\prime}\right)$ [14] is given by;

$$
\Psi\left(\mathrm{r}, \mathrm{r}^{\prime}, \tilde{k}\right)=\int_{V} G\left(\mathrm{r}, \mathrm{r}^{\prime}, \tilde{k}\right) F\left(r^{\prime}\right) d^{3} r^{\prime}
$$

For the system with $y<<k$, the Green's function can further be expressed with the Eigen modes [14] given as; 


$$
G\left(\mathrm{r}, \mathrm{r}^{\prime}, \tilde{k}\right)=\sum_{n} \frac{\phi_{n}^{*}\left(r^{\prime}\right) \cdot \phi_{n}(r)}{\left(k^{2}-\mathrm{k}_{n}^{2}\right)+2 i y k^{\prime}}
$$

Where, $\phi_{n}(r)$ and $k_{n}$ are the eigenmodes and eigenvalues. Substituting Equation (5) into Equation (4), the normalized response function $\Psi\left(r, r^{\prime}, \tilde{k}\right)$ is given by Equation (6);

$$
\Psi\left(r, r^{\prime}, \tilde{k}\right)=\sum_{n} a_{n}\left(r^{\prime}, \tilde{k}\right) \phi_{n}(r)
$$

Considering a piece of square plate, the eigenmodes are given by Equation (7);

$$
\phi_{n_{1}, n_{2}}(x, y)=\frac{2}{a} \cos \left(\frac{n_{1} \pi}{a} x\right) \cos \left(\frac{n_{2}}{a}\right) y
$$

Where $\mathrm{n}_{1}=0,1,2,3 \ldots$ and $\mathrm{n}_{2}=0,1,2,3 \ldots .$. , the eigenvalues corresponding to the eigenmodes $\phi_{n_{1}, n_{2}}(x, y)$ are given Equation (8);

$$
k_{n_{1}, n_{2}}=\frac{\pi}{a} \sqrt{n_{1}^{2}+} n_{2}^{2}
$$

With a point source at the centre, the driving function can be expressed in Equation (9);

$$
F\left(r^{\prime}\right)=F_{o} \delta\left(x^{\prime}-a / 2\right) \delta\left(y^{\prime}-a / 2\right)
$$

Where, $F_{0}$ is the amplitude of the driving source Equation (10) is the forth-order wave equation describing flexural or bending waves in the thin plate vibrating under the influence of electro-mechanical oscillator [15].

$\rho t \frac{\partial^{2} u}{\partial t^{2}}+B_{x x} \frac{\partial^{4} u}{\partial x^{4}}+2 B_{x y} \frac{\partial^{2} u}{\partial x^{2} \partial y^{2}}+B_{y y} \frac{\partial^{4} u}{\partial y^{4}}=0$

Where,

$$
B_{x x}=\frac{E_{x x} t^{3}}{12 \rho\left(1-v_{x x}^{2}\right)} \text { and } B_{y y}=\frac{E_{y y} t^{3}}{12 \rho\left(1-v_{y y}^{2}\right)}
$$

Where, $\mathrm{t}$ is the flat plate thickness, $\mathrm{E}_{\mathrm{xx}}, v_{y y}, \mathrm{~B}_{\mathrm{xx}}$ and $B_{y y}$ are the elastic constant along the symmetrical regions in the in the $x$ and $y$ axis of the square/rectangular plates.

For thin rectangular/square plates under the influence of vibration, Kaczmarek et al. [16] proposed a relationship for the frequency of modes $(\mathrm{Hz})$ expressed in Equation (12);

$$
f=\sqrt{\left(\frac{n}{l}\right)^{2}+\left(\frac{m}{w}\right)^{2}}
$$

Where, /is length of the plate, $w$ is width of the plate

\section{RESULTS AND DISCUSSION}

As shown earlier in Figure $1 \mathrm{a}$, a circular region of the oscillator can be seen on the surface of the vibrator when the square-like flat plate is not yet installed. When the electromagnetic oscillator was connected to a power source and the square plate properly positioned on top of the circular part, the vibrating effects of the oscillator at different frequencies were transmitted through the circular region of the oscillator in Figure $1 \mathrm{a}$, to the $0.4 \mathrm{~mm} \times 0.40 \mathrm{~mm}$ thin square plate which generated various patterns in the process. Figure 3 represents the traveling wave on the circular region of the oscillator, resulting from the vibrating effects at different frequencies. Tables 1-4 represent the frequency values obtained from the experimental analysis and FEM solvers, while Figures 4-11 show the plot for each time interval.

A thin plate (with fine grains on the surface) subjected to vibration can produce several different vibration mode shapes, each with a different pattern of nodal lines as shown in Figures 12-15.

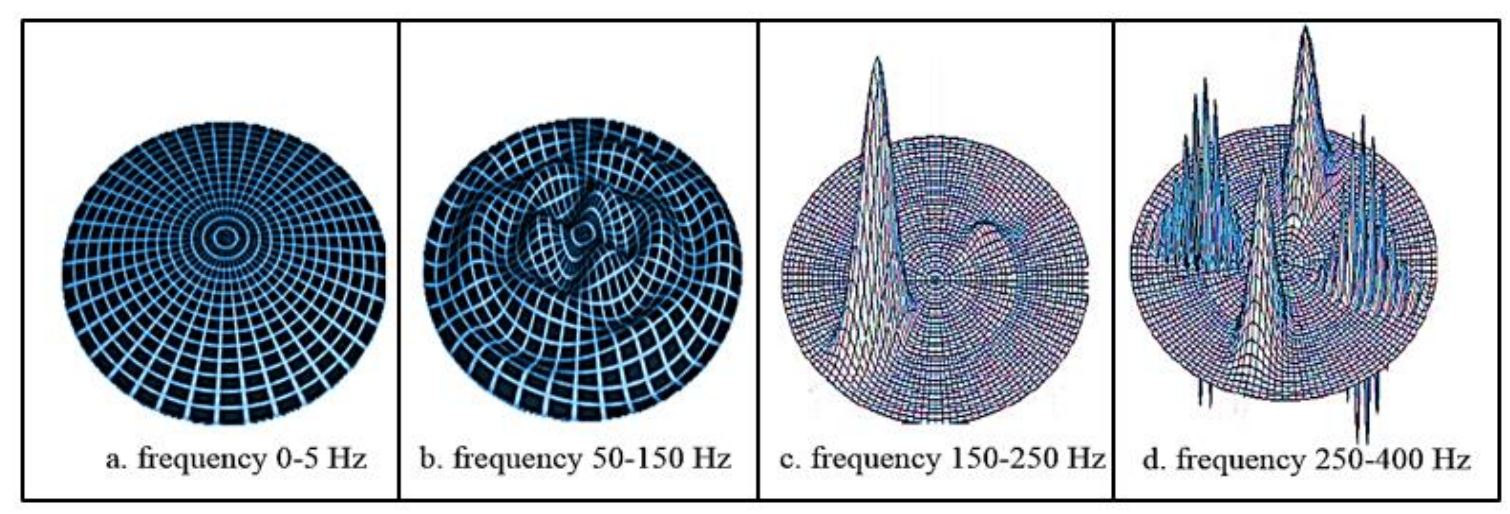

Figure 3: Vibration Effects on the Circular Surface Region of the Oscillator at different Frequencies

Table 1: Results of Modal Frequency Obtain from the Experimental Procedure

\begin{tabular}{cccccccccc}
\hline Steps & Time $(\mathrm{s})$ & \multicolumn{7}{c}{ Modal Frequencies $(\mathrm{Hz})$} & \\
\hline 1. & 7 & 100 & 100 & 100 & 100 & 100 & 100 & 100 & 100 \\
2. & 14 & 113 & 115 & 116 & 115 & 118 & 115 & 118 & 112 \\
3. & 21 & 138 & 140 & 143 & 140 & 138 & 135 & 133 & 136 \\
4. & 28 & 164 & 167 & 162 & 164 & 161 & 167 & 165 & 163 \\
\hline
\end{tabular}


Response Variation of Chladni Patterns on Vibrating Elastic Plate under Electro-Mechanical Oscillation, A. E. Ikpe, et. al

\begin{tabular}{cccccccccc}
\hline Steps & Time $(\mathrm{s})$ & \multicolumn{7}{c}{ Modal Frequencies $(\mathrm{Hz})$} \\
\hline 5. & 35 & 185 & 176 & 183 & 189 & 192 & 188 & 184 & 187 \\
6. & 42 & 202 & 207 & 209 & 214 & 216 & 218 & 213 & 212 \\
7. & 49 & 218 & 224 & 226 & 230 & 228 & 234 & 225 & 223 \\
8. & 56 & 226 & 232 & 234 & 239 & 243 & 245 & 236 & 240 \\
\hline
\end{tabular}

Table 2: Results of Modal Frequency Obtain from HYPERMESH Solver

\begin{tabular}{cccccccccc}
\hline Steps & Time $(\mathrm{s})$ & \multicolumn{7}{c}{ Modal Frequencies $(\mathrm{Hz})$} \\
\hline 1. & 7 & 100 & 100 & 100 & 100 & 100 & 100 & 100 & 100 \\
2. & 14 & 112 & 115 & 114 & 115 & 118 & 116 & 117 & 113 \\
3. & 21 & 136 & 141 & 143 & 142 & 137 & 134 & 130 & 134 \\
4. & 28 & 166 & 165 & 163 & 165 & 161 & 166 & 164 & 163 \\
5. & 35 & 186 & 177 & 180 & 188 & 194 & 188 & 185 & 187 \\
6. & 42 & 204 & 209 & 210 & 215 & 214 & 220 & 215 & 209 \\
7. & 49 & 215 & 223 & 227 & 232 & 229 & 234 & 227 & 223 \\
8. & 56 & 227 & 230 & 233 & 241 & 242 & 244 & 236 & 239 \\
\hline
\end{tabular}

Table 3: Results of Modal Frequency Obtain from ANSYS R15.0 Solver

\begin{tabular}{cccccccccc}
\hline Steps & Time $(\mathrm{s})$ & \multicolumn{7}{c}{ Modal Frequencies $(\mathrm{Hz})$} \\
\hline 1. & 7 & 100 & 100 & 100 & 100 & 100 & 100 & 100 & 100 \\
2. & 14 & 107 & 110 & 109 & 108 & 112 & 115 & 118 & 114 \\
3. & 21 & 136 & 125 & 137 & 138 & 128 & 127 & 127 & 134 \\
4. & 28 & 161 & 158 & 164 & 153 & 157 & 158 & 156 & 168 \\
5. & 35 & 182 & 172 & 176 & 184 & 190 & 186 & 190 & 192 \\
6. & 42 & 207 & 206 & 205 & 215 & 217 & 229 & 221 & 211 \\
7. & 49 & 218 & 218 & 227 & 236 & 233 & 235 & 232 & 230 \\
8. & 56 & 233 & 232 & 239 & 242 & 240 & 241 & 242 & 238 \\
\hline
\end{tabular}

Table 4: Results of Modal Frequency Obtain from CATIA Solver

\begin{tabular}{cccccccccc}
\hline Steps & Time $(\mathrm{s})$ & \multicolumn{7}{c}{ Modal Frequencies $(\mathrm{Hz})$} & \\
\hline 1. & 7 & 100 & 100 & 100 & 100 & 100 & 100 & 100 & 100 \\
2. & 14 & 118 & 117 & 121 & 115 & 114 & 112 & 113 & 110 \\
3. & 21 & 135 & 146 & 137 & 138 & 135 & 132 & 138 & 134 \\
4. & 28 & 167 & 162 & 156 & 166 & 166 & 163 & 167 & 160 \\
5. & 35 & 186 & 179 & 187 & 177 & 197 & 184 & 182 & 183 \\
6. & 42 & 207 & 203 & 215 & 216 & 218 & 222 & 214 & 214 \\
7. & 49 & 217 & 228 & 229 & 232 & 230 & 231 & 227 & 223 \\
8. & 56 & 230 & 235 & 237 & 241 & 242 & 240 & 238 & 236 \\
\hline
\end{tabular}

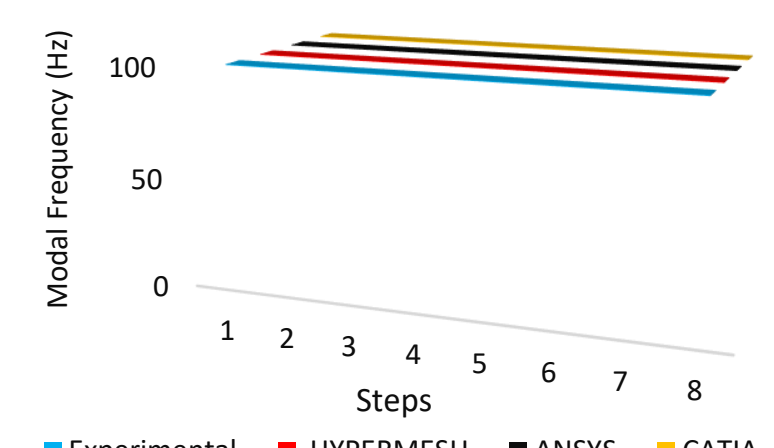

Experimental $\square$ HYPERMESH $\square$ ANSYS $\square$ CATIA

Figure 4: Modal Frequency at 7s

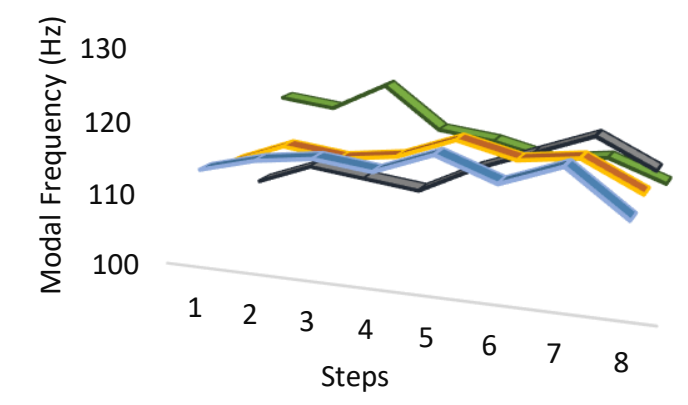

Experimental $\square$ HYPERMESH $\square$ ANSYS $\square$ CATIA

Figure 5: Modal Frequency at 14s 


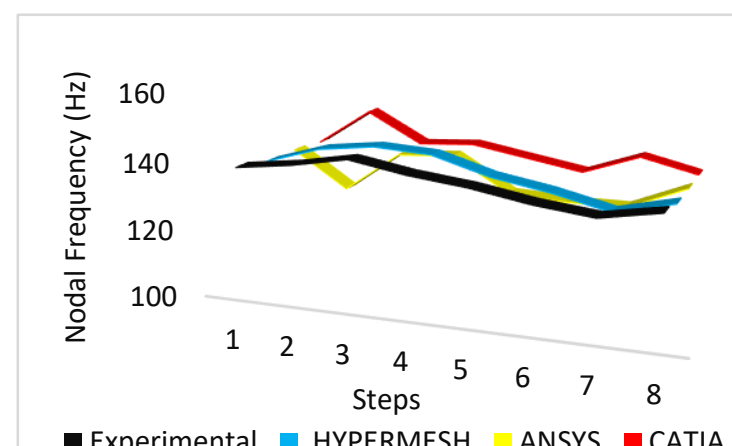

- Experimental $\square$ HYPERMESH ANSYS —CATIA

Figure 6: Modal Frequency at 21s

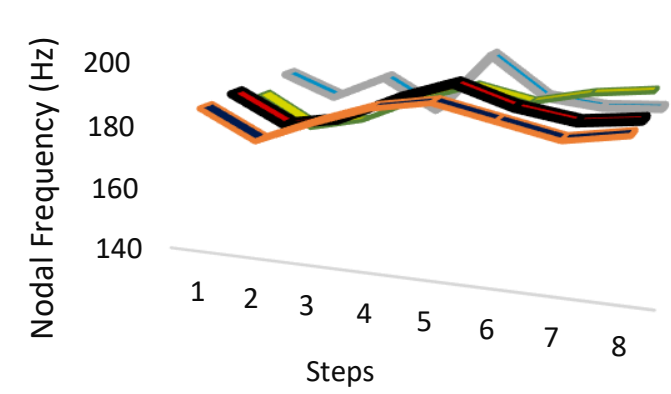

—Experimental $\square$ HYPERMESH $\square$ ANSYS $\square$ CATIA

Figure 8: Modal Frequency at 35s

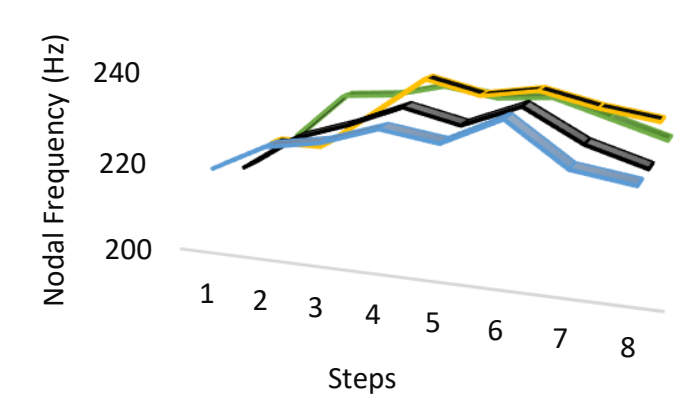

$\square$ Experimental $\square$ HYPERMESH $\square$ ANSYS $\square$ CATIA

Figure 10: Modal Frequency at 49s
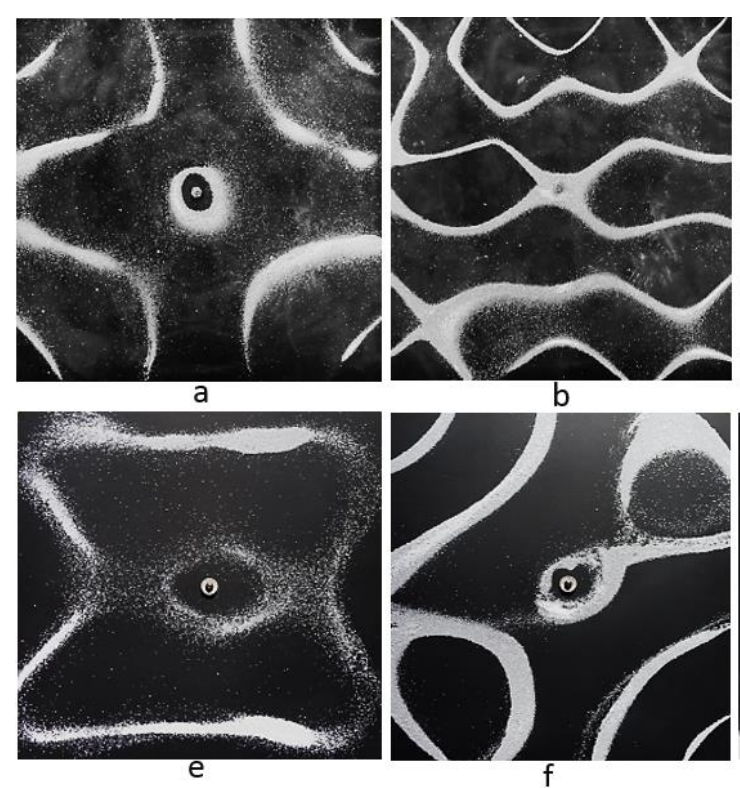

Figure 12: Chladni Patterns Obtain from the Experimental Procedure

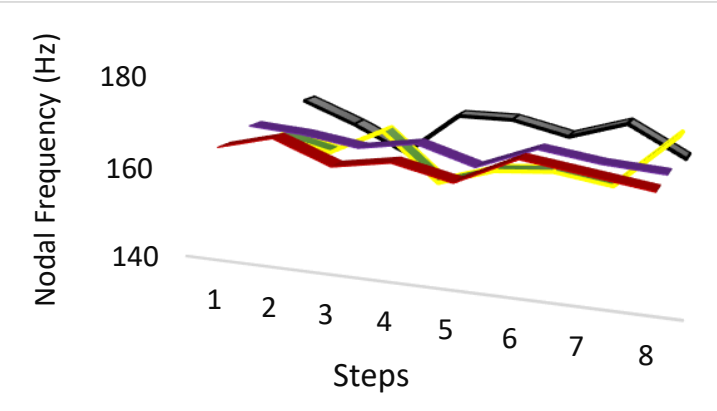

$\square$ Experimental $\square$ HYPERMESH $\square$ ANSYS $\square$ CATIA

Figure 7: Modal Frequency at 28s

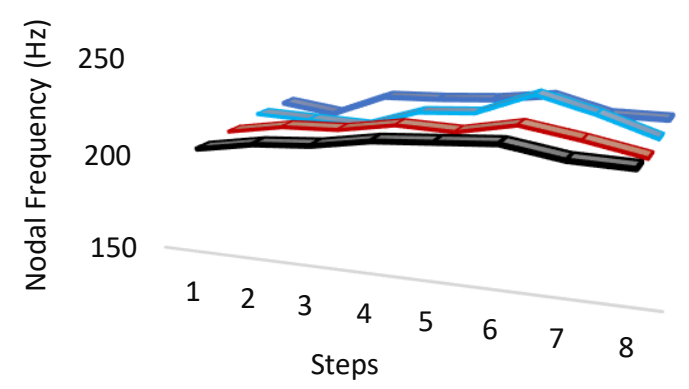

口Experimental $\square$ HYPERMESH $\square$ ANSYS $\square$ CATIA

Figure 9: Modal Frequency at 42s

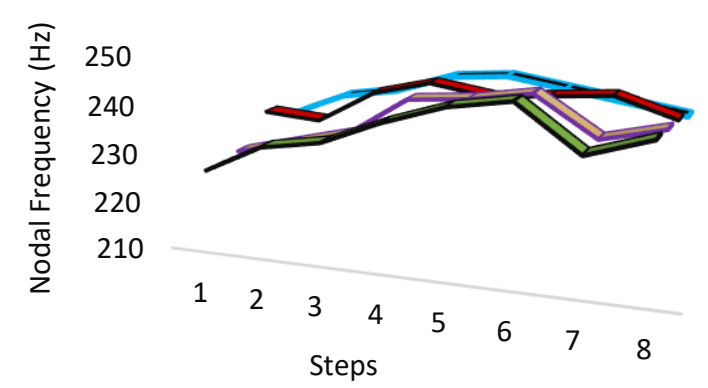

$\square$ Experimental $\square$ HYPERMESH $\square$ ANSYS $\square$ CATIA

Figure 11: Modal Frequency at 56s
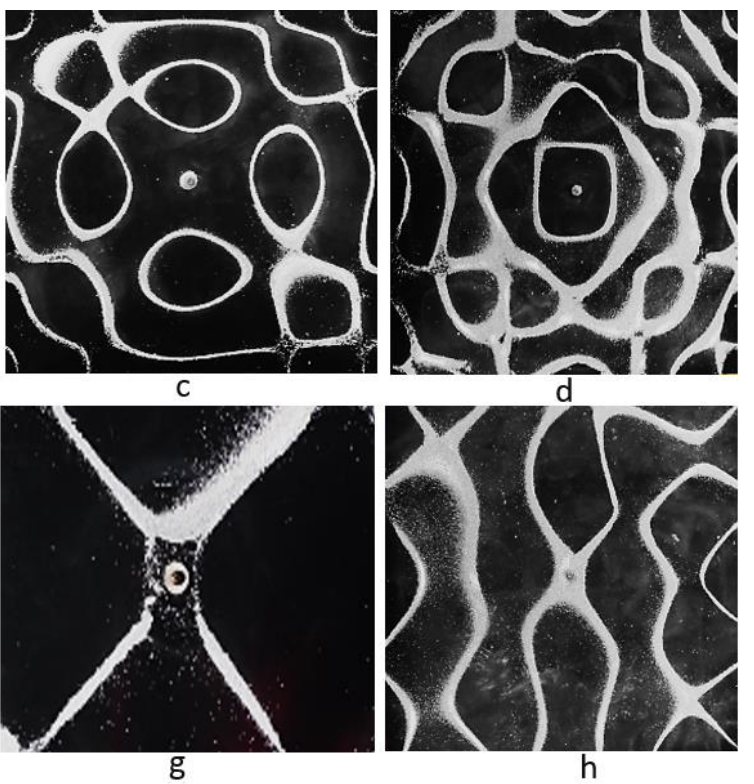

July 2019 


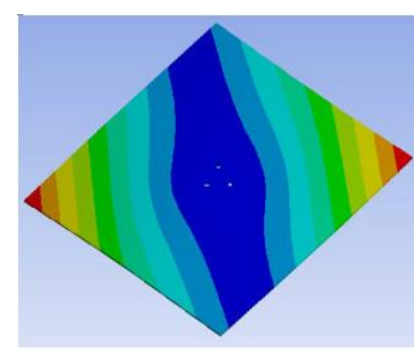

a

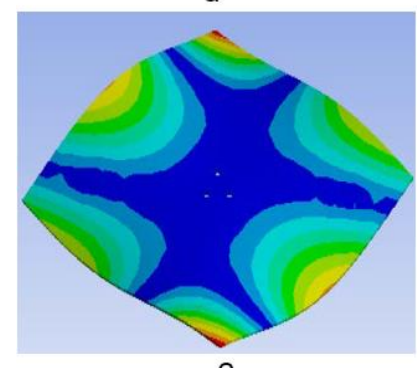

e

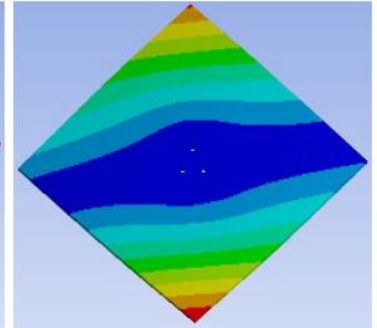

b

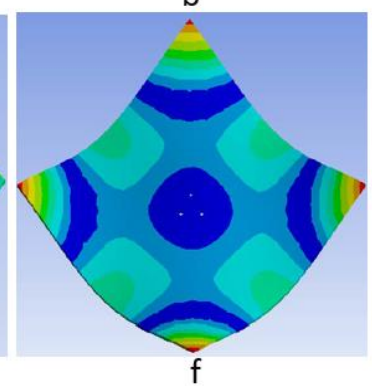

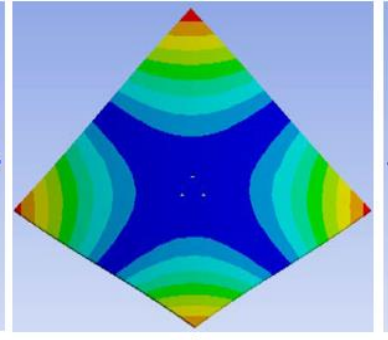

C

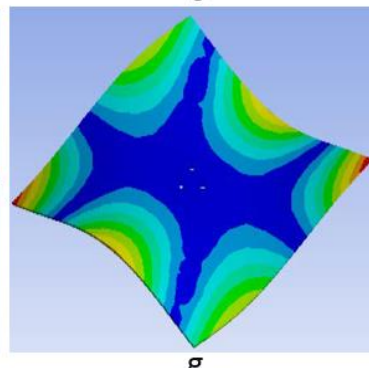

$\mathrm{g}$

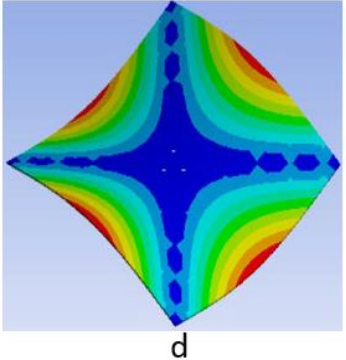

d

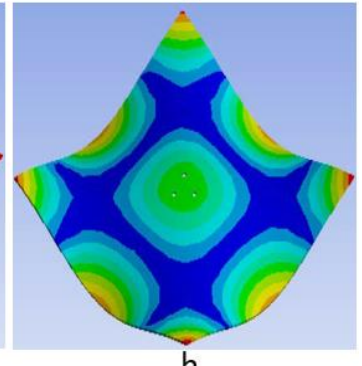

Figure 13: Chladni Patterns Obtain from HYPERMESH Solver
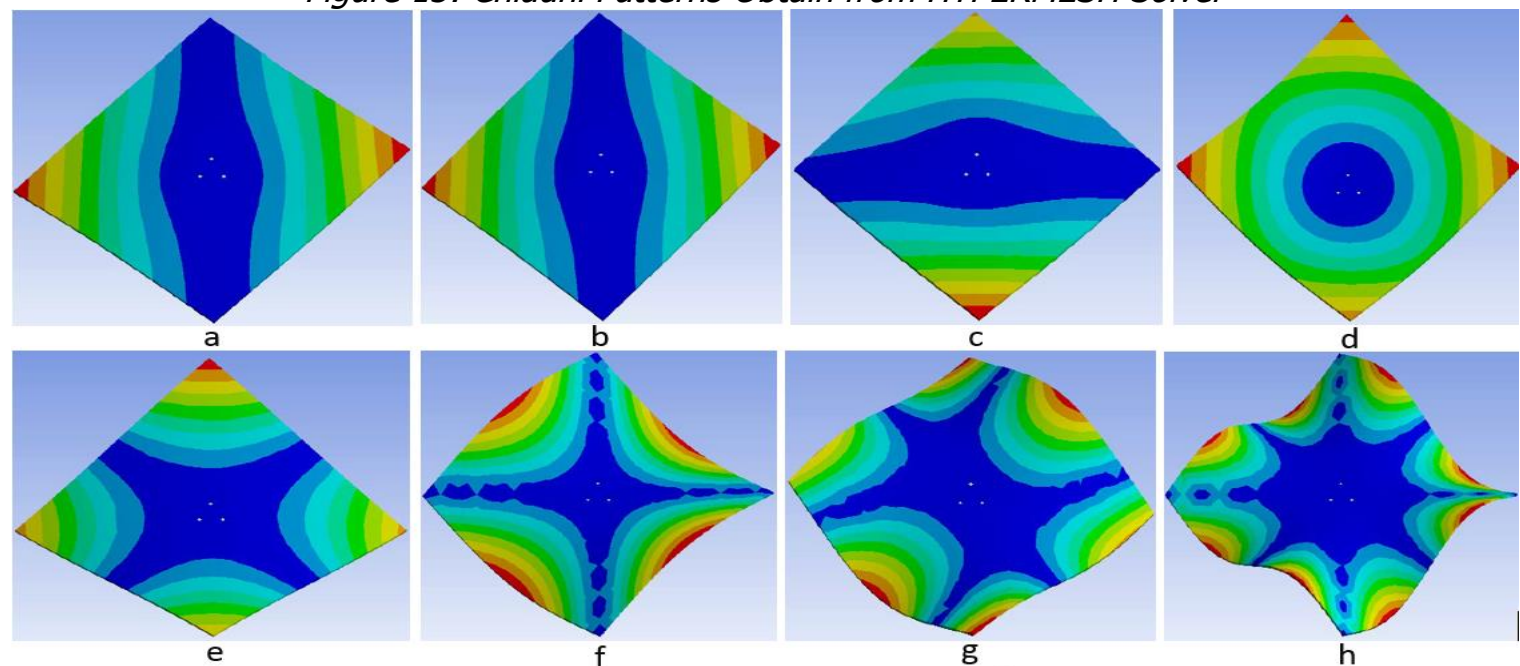

Figure 14: Chladni Patterns Obtain from ANSYS R15.0 Solver
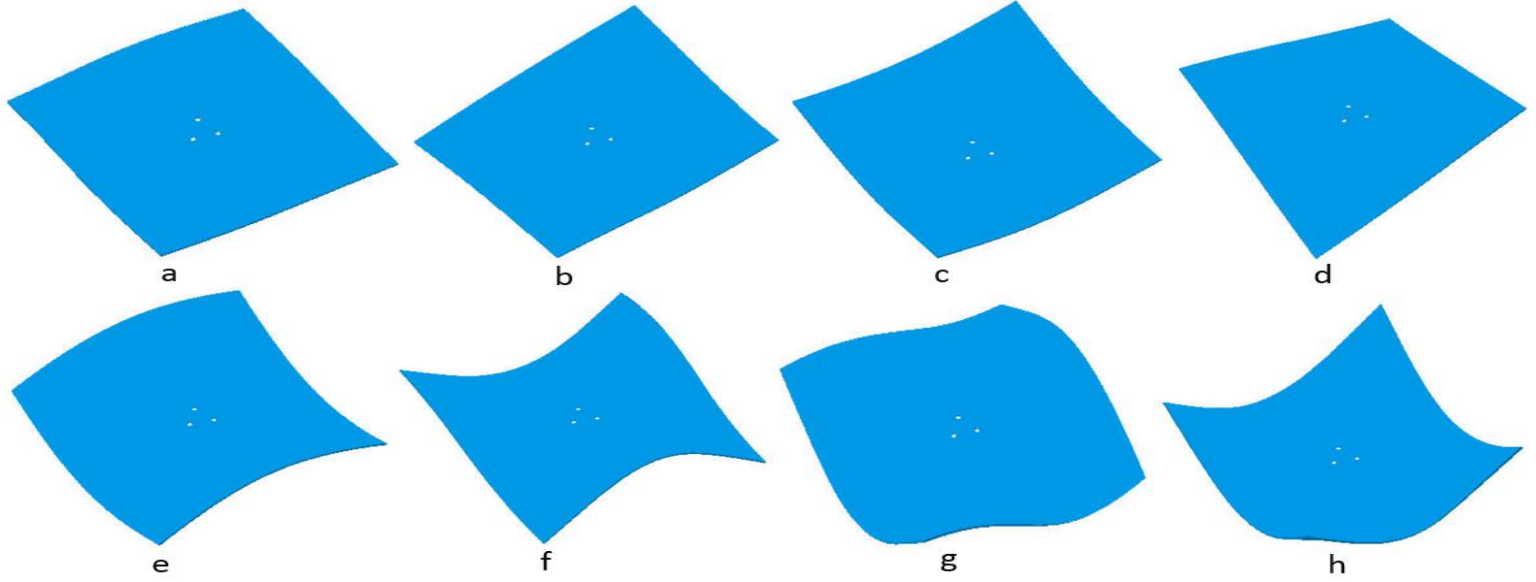

Figure 15: Chladni Patterns Obtain from CATIA Solver

Finite Element Method (FEM) is a useful tool that has become relevant in numerical, statistical and in most complex problems that human capacity would barely unravel. For example, the application of three finite element solvers in this study (CATIA 2017 version, ANSYS R15.0 2017 version and HYPERMESH 2016 version) have helped unravel the complexities surrounding the theory of Chladni patterns. From the graphical representation (see Figure 4-11) of modal frequencies obtained from experimentally and through the use of FEM, frequency values obtained from HYPERMESH solver is observed to be the closest 
to the modal frequencies obtained experimentally. This agrees with the investigation carried out by Owunna et al. [7] on experimental modal analysis of a flat plate subjected to vibration. The experimental investigation in this study was designed to show the influence of a plate geometry on the modal shapes (the shapes characterized by line patterns appear as the frequencies resonate with the thin plate) formed when the plate is subjected to vibration (the overlap of the waves results an interference pattern of nodes). Therefore, a thin sheet of metal excited at resonance is divided into various patterns vibrating in opposite directions bounded by lines of vibration referred to as nodal lines. The visibility of these nodal lines was achieved by sprinkling sugar on the surface of the excited thin plate under vibration. The various positions on the surface of the plate where the sugar particles bunched up and appeared to halt in motion are known as the nodes. In other words, as the frequency varies, the position of the nodes adjust gradually until they stagnate at a point where fine imaginary lines patterns are formed [17]. By so doing, the sugar particles skitter from one end of the plate to the other and bunches up at a point, transforming itself into a more complex geometric shapes. In the experimental process, it was observed that as the frequency varied, the position of the nodes adjusted across the top plane of the plate. It was also observed that the longer the excitation time, the higher the modal frequencies and the more complex the shapes and patterns formed on the plate surface as shown in Tables 1-4 and Figures 12-15. In this case, higher frequencies imply more peaks in the sound wave, and thus increasing nodes in the resulting interference pattern. Therefore, as the sound waves resonate through the thin metal sheet, there is a backward reflection of the sound towards the source, producing a sound that irritates the ear. In recent times, loud speaker and electronic signal generator such as the electromagnetic single axis systems have been employed to control the frequency of the sound as it increases. It should be noted that the line patterns, mode shapes as well as the frequencies obtained in this study is only for square plate, as the use of circular, triangular and rectangular plates will provide great variety of patterns different from those presented in this study. Figure $16 \mathrm{a}$ represent the forces experienced by particles with high damping coefficients while Figure $16 \mathrm{~b}$ represent the forces experienced by particles with low damping coefficients.

The resulting velocity of a grain particle bouncing on the surface of a vibrating plate depends upon the velocity of the plate upon impact, particle velocity before collision, and the viscous damping coefficient. Rise in the viscous damping coefficient proportionally decreases the resulting particle velocity by absorbing more of the force applied on the vibrating plate. However, in cases where the damping force is sufficient enough such that the breakoff force exceeds the applied force, then the particle will not bounce at all, and will rather stick to the plate surface in a short period of time until the force exerted by the plate exceeds the breakoff force including the effect of viscous damping. This agrees with the investigation carried out by Shridhar [9], and plays a vital role in any successful experiment on acoustically excited plate.
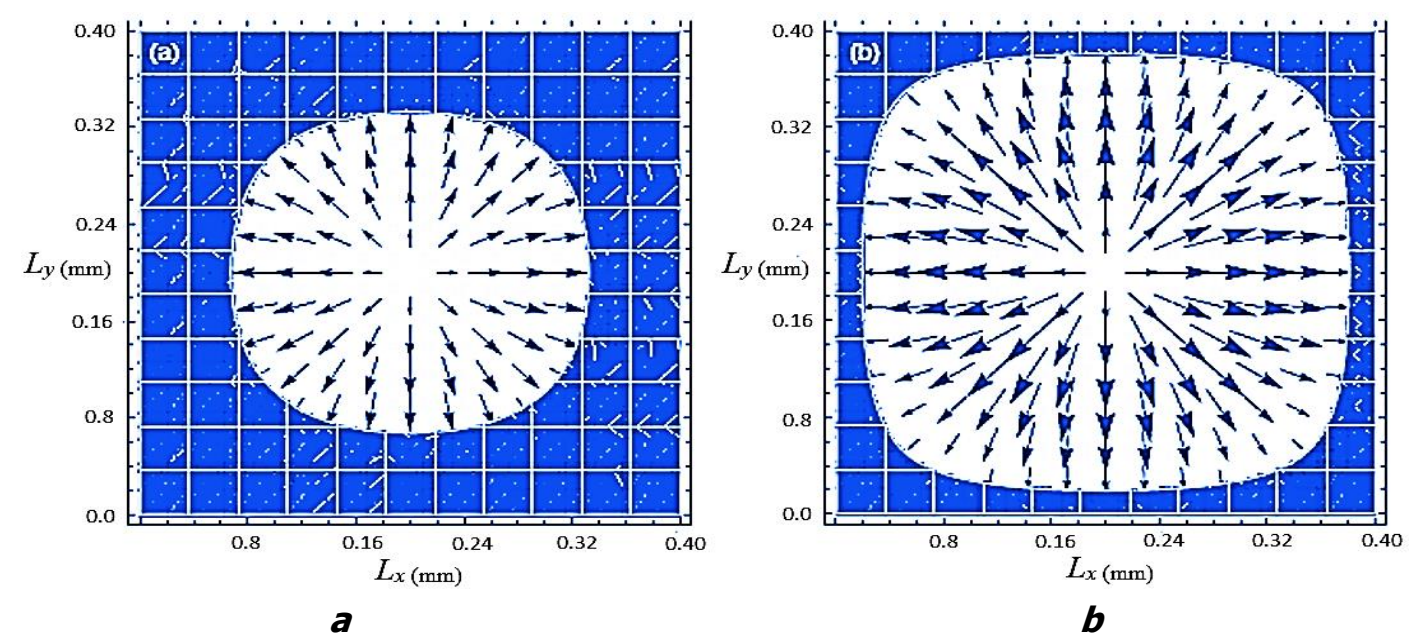

Figure 16: Effect of Particle Damping on the Force Exerted on a Bouncing Particle on a Vibrating Plate 


\section{CONCLUSION}

Finite Element Method has been successfully employed in this study to emulate the modal frequencies and patterns in thin plates under acoustic excitation, and the results obtained correlates with the experimental values. This can serve as alternative to the numerical and experimental methods, considering the proximity between the experimental values and FEM values. For further investigation in engineering field, FEM can be adopted to check the effects of acoustic excitations on displacement, deformation and stress profiles of thin plates. This could unravel the challenges surrounding the stress build-ups in mechanical and structural components in relation to their failure mechanisms.

\section{REFERENCES}

[1] Ullmann, D. (2007) Life and work of e.f.f. chladni. The European Physical Journal-Special Topics, 145:27.

[2] Gough, C. (2007) The violin: Chladni patterns, plates, shells and sounds. The European Physical Journal - Special Topics, 145:77,101, 2007.

[3] Lindemann, T. and Vella, A. (2015) Analysis of Vibrating Plates with Acoustic Holography and Eddy Currents. University of Illinois, USA.

[4] Arango, J. and Reyes, C. (2015) Stochastic Models for Chladni Figures. Proceedings of the Edinburgh Mathematical Society, 59(2), 287-300.

[5] Trejo-Mandujano, H. A., Mijares-Bernal, G. and Ordoneez-Casanova, E. G. (2015) Alternate Model of Chladni Figures for the Circular Homogeneous Thin Plate Case with Open Boundaries. Journal of Physics: Conference Series, 582,012022.

[6] Amore, P. (2008) Solving the Helmholtz Equation for Membranes of Arbitrary Shape: Numerical Result. Journal of Physics A: Mathematical and Theoretical, 41(26), 1-32.

[7] Owunna, I., Ikpe, A., Satope, P. and Ikpe, E. (2016) Experimental Modal Analysis of a Flat Plate Subjected To Vibration. American Journal of Engineering Research, 5(6), 30-37.
[8] Muller, T. (2013) Numerical Chladni figures. University of Stuttgart, Allmandring 19, 70569 Stuttgart, Germany.

[9] Shridhar, M. (2011) Manipulating Standard and Inverse Chladni Patterns by Modulating Adhesive, Frictional, and Damping Forces. International Journal of Scientific and Engineering Research, 3(5), 782789.

[10] Zhou, Q., Sariola, V., Latifi, K. and Liimatainen, V. (2016) Controlling the motion of multiple objects on a Chladni plate. Nature Communications, 7(12764), 1-10.

[11] Vuillermet, G. (2016) Chladni Patterns in a Liquid at Microscale. American Physical Society, Physical Review Letters, 116, 184501-5.

[12] Mama, B. O., Onah, H. N., Ike, C. C. and Osadebe, N. N. (2017) Solution of Free Harmonic Vibration Equation of Simply Supported Kirchhooff Plate by Galerkin-Vlasov Method. Nigerian Journal of Technology, 36(2), 361-365.

[13] Blevins, R. D. (1979) Formulas for Natural Frequencies and Mode Shapes. Van NostrandReinhold Co, USA, ISBN: 9780442207106.

[14] Tuan, H., Wen, C. P., Chiang, P. Y. and Yu, Y. T. (2015) Exploring the Resonant Vibration of Thin Plate: Reconstruction of Chladni Patterns and Determination of Resonant Wave Numbers. The Journal of the Acoustical Society of America 137 (4) 2113-2123.

[15] Cuenca, J. (2009) Wave Models for the Flexural Vibrations of Thin Plates-Model of the Vibrations of Polygonal Plates by the Image Source Method-Vibration Damping Using the Acoustic Black Hole Effect. University of Maine, USA, tel-00442260.

[16] Kaczmarek, A., Javorek, L. And Orłowski, K. (2014) Mode Vibrations of Plates-Experimental Analysis Annals of Warsaw University of Life Sciences, Forestry and Wood Technology 88 97101.

[17] Skrodzki, M. Reitebuch, U. and Polthier, K. (2016) Chladni Figures Revisited: A Peek into the Third Dimension, in Proceedings of Bridges Finland Conference: 2016, 481-484 